\title{
Non-Newtonian polytropic filtration systems with nonlinear boundary conditions
}

Wanjuan Du* and Zhongping Li

* Correspondence:

duwanjuan28@163.com

College of Mathematic and

Information, China West Normal University, Nanchong 637002, PR China

\section{Abstract}

This article deals with the global existence and the blow-up of non-Newtonian polytropic filtration systems with nonlinear boundary conditions. Necessary and sufficient conditions on the global existence of all positive (weak) solutions are obtained by constructing various upper and lower solutions.

Mathematics Subject Classification (2000)

$35 \mathrm{~K} 50,35 \mathrm{~K} 55,35 \mathrm{~K} 65$

Keywords: Polytropic filtration systems, Nonlinear boundary conditions, Global existence, Blow-up

\section{Introduction}

In this article, we study the global existence and the blow-up of non-Newtonian polytropic filtration systems with nonlinear boundary conditions

$$
\begin{array}{ll}
\left(u_{i}^{k_{i}}\right)_{t}=\Delta_{m_{i}} u_{i}(i=1, \ldots, n), & x \in \Omega, t>0, \\
\nabla_{m_{i}} u_{i} \cdot v=\prod_{j=1}^{n} u_{j}^{m_{i j}}(i=1, \ldots, n), & x \in \partial \Omega, t>0, \\
u_{i}(x, 0)=u_{i 0}(x)>0(i=1, \ldots, n), & x \in \bar{\Omega},
\end{array}
$$

where

$$
\Delta_{m_{i}} u_{i}=\operatorname{div}\left(\left|\nabla u_{i}\right|^{m_{i}-1} \nabla u_{i}\right)=\sum_{j=1}^{N}\left(\left|\nabla u_{i}\right|^{m_{i}-1} u_{i x_{j}}\right)_{x_{j}{ }^{\prime}} \nabla_{m_{i}} u_{i}=\left(\left|\nabla u_{i}\right|^{m_{i}-1} u_{i x_{1}}, \ldots,\left|\nabla u_{i}\right|^{m_{i}-1} u_{i x_{N}}\right),
$$

$\Omega \subset \mathbb{R}^{N}$ is a bounded domain with smooth boundary $\partial \Omega, v$ is the outward normal vector on the boundary $\partial \Omega$, and the constants $k_{i}, m_{i}>0, m_{i j} \geq 0, i, j=1, \ldots, n ; u_{i 0}(x)(i$ $=1, \ldots, n)$ are positive $C^{1}$ functions, satisfying the compatibility conditions.

The particular feature of the equations in (1.1) is their power- and gradient-dependent diffusibility. Such equations arise in some physical models, such as population dynamics, chemical reactions, heat transfer, and so on. In particular, equations in (1.1) may be used to describe the nonstationary flows in a porous medium of fluids with a power dependence of the tangential stress on the velocity of displacement under polytropic conditions. In this case, the equations in (1.1) are called the non-Newtonian polytropic filtration equations which have been intensively studied (see [1-4] and the references therein). For the Neuman problem (1.1), the local existence of solutions in time have been established; see the monograph [4].

(c) 2011 Du and Li; licensee Springer. This is an Open Access article distributed under the terms of the Creative Commons Attribution License (http://creativecommons.org/licenses/by/2.0), which permits unrestricted use, distribution, and reproduction in any medium provided the original work is properly cited. 
We note that most previous works deal with special cases of (1.1) (see [5-13]). For example, Sun and Wang [7] studied system (1.1) with $n=1$ (the single-equation case) and showed that all positive (weak) solutions of (1.1) exist globally if and only if $m_{11} \leq$ $k_{1}$ when $k_{1} \leq m_{1}$; and exist globally if and only if $m_{11} \leq \frac{m_{1}\left(k_{1}+1\right)}{m_{1}+1}$ when $k_{1}>m_{1}$. In [13], Wang studied the case $n=2$ of (1.1) in one dimension. Recently, Li et al. [5] extended the results of [13] into more general $\mathrm{N}$-dimensional domain.

On the other hand, for systems involving more than two equations when $m_{i}=1(i=$ $1, \ldots, n)$, the special case $k_{i}=1(i=1, \ldots, n)$ (heat equations) is concerned by Wang and Wang [9], and the case $k_{i} \leq 1(i=1, \ldots, n)$ (porous medium equations) is discussed in [12]. In both studies, they obtained the necessary and sufficient conditions to the global existence of solutions. The fast-slow diffusion equations (there exists $i(i=1, \ldots, n)$ such that $k_{i}>1$ ) is studied by Qi et al. [6], and they obtained the necessary and sufficient blow up conditions for the special case $\Omega=B_{R}(0)$ (the ball centered at the origin in $\mathbb{R}^{N}$ with radius $R$ ). However, for the general domain $\Omega$, they only gave some sufficient conditions to the global existence and the blow-up of solutions.

The aim of this article is to study the long-time behavior of solutions to systems (1.1) and provide a simple criterion of the classification of global existence and nonexistence of solutions for general powers $k_{i} m_{i}$, indices $m_{i j}$, and number $n$.

Define

$$
\begin{aligned}
b_{i} & =\min \left\{k_{i}, \frac{m_{i}\left(k_{i}+1\right)}{m_{i}+1}\right\}, \quad b_{i j}=b_{i} \delta_{i j}, \quad i, j=1, . ., n, \\
B & =\left(b_{i j}\right)_{n \times n}, \quad M=\left(m_{i j}\right)_{n \times n}, \quad A=B-M .
\end{aligned}
$$

Our main result is

Theorem. All positive solutions of (1.1) exist globally if and only if all of the principal minor determinants of $A$ are non-negative.

Remark. The conclusion of Theorem covers the results of [5-13]. Moreover, this article provides the necessary and sufficient conditions to the global existence and the blow-up of solutions in the general domain $\Omega$. Therefore, this article improves the results of [6].

The rest of this article is organized as follows. Some preliminaries will be given in next section. The above theorem will be proved in Section 3 .

\section{Preliminaries}

As is well known that degenerate and singular equations need not possess classical solutions, we give a precise definition of a weak solution to (1.1).

Definition. Let $T>0$ and $Q_{T}=\Omega \times(0, T]$. A vector function $\left(u_{1}(x, t), . ., u_{n}(x, t)\right)$ is called a weak upper (or lower) solution to (1.1) in $Q_{T}$ if

(i). $u_{i}(x, t)(i=1, \ldots, n) \in L^{\infty}\left(0, T ; W^{1, \infty}(\Omega)\right) \cap W^{1,2}\left(0, T ; L^{2}(\Omega)\right) \cap C\left(\overline{Q_{T}}\right) ;$

(ii). $\left(u_{1}(x, 0), \ldots, u_{n}(x, 0)\right) \geq(\leq)\left(u_{10}(x), \ldots, u_{n 0}(x)\right)$;

(iii). for any positive functions $\psi_{i}(i=1, \ldots, n) \in L^{1}\left(0, T ; W^{1,2}(\Omega)\right) \cap L^{2}\left(Q_{T}\right)$, we have

$$
\iint_{Q_{T}}\left[\left(u_{i}^{k_{i}}\right)_{t} \psi_{i}+\nabla_{m_{i}} u_{i} \cdot \nabla \psi_{i}\right] d x d t \geq(\leq) \int_{0}^{T} \int_{\partial \Omega} \prod_{j=1}^{n} u_{j}^{m_{i j}} \psi_{i} d s d t \quad(i=1, \ldots, n) .
$$

In particular, $\left(u_{1}(x, t), \ldots, u_{n}(x, t)\right)$ is called a weak solution of (1.1) if it is both a weak upper and a lower solution. For every $T<\infty$, if $\left(u_{1}(x, t), \ldots, u_{n}(x, t)\right)$ is a solution of (1.1) in $Q_{T}$, then we say that $\left(u_{1}(x, t), \ldots, u_{n}(x, t)\right)$ is global. 
Lemma 2.1 (Comparison Principle.) Assume that $u_{i 0}(i=1, \ldots, n)$ are positive $C^{1}(\bar{\Omega})$ functions and $\left(u_{1}, \ldots, u_{n}\right)$ is any weak solution of $(1.1)$. Also assume that $\left(\underline{u}_{1}, \ldots, \underline{u}_{n}\right)$ $\geq(\delta, \ldots, \delta)>0$ and $\left(\bar{u}_{1}, \ldots, \bar{u}_{n}\right)$ are the lower and upper solutions of (1.1) in $Q_{T}$, respectively, with nonlinear boundary flux $\quad\left(\underline{\lambda} \prod_{j=1}^{n} \underline{u}_{j}^{m_{1 j}}, \ldots, \lambda \prod_{j=1}^{n} \underline{u}_{j}^{m_{n j}}\right)_{a n d}$ $\left(\bar{\lambda} \prod_{j=1}^{n} \bar{u}_{j}^{m_{1 j}}, \ldots, \bar{\lambda} \prod_{j=1}^{n} \bar{u}_{j}^{m_{n j}}\right)$, where $0<\underline{\lambda}<1<\bar{\lambda}$. Then we have $\left(\bar{u}_{1}, \ldots, \bar{u}_{n}\right) \geq\left(u_{1}, \ldots, u_{n}\right) \geq\left(\underline{u}_{1}, \ldots, \underline{u}_{n}\right)_{\text {in }} Q_{T}$.

When $n=2$, the proof of Lemma 2.1 is given in [5]. When $n>2$, the proof is similar.

For convenience, we denote $0<\underline{\lambda}<1<\bar{\lambda}$, which are fixed constants, and let $\delta=\min _{1 \leq i \leq n}\left\{\min _{\bar{\Omega}} u_{i 0}(x)\right\}>0$.

In the following, we describe three lemmas, which can be obtained directly from Lemmas 2.7-2.9 in [6].

Lemma 2.2 Suppose all the principal minor determinants of $A$ are non-negative. If $A$ is irreducible, then for any positive constant $c$, there exists $\alpha=\left(\alpha_{1}, \ldots, \alpha_{n}\right)^{T}$ such that $A$ $\alpha \geq 0$ and $\alpha_{i}>c(i=1, \ldots, n)$.

Lemma 2.3 Suppose that all the lower-order principal minor determinants of $A$ are non-negative and $A$ is irreducible. For any positive constant $C$, there exist large positive constants $L_{i}(i=1, \ldots, n)$ such that

$$
\prod_{j=1}^{n} L_{j}^{a_{i j}} \geq C \quad(i=1, \ldots, n) .
$$

Lemma 2.4 Suppose that all the lower-order principal minor determinants of $A$ are non-negative and $|A|<0$. Then, $A$ is irreducible and, for any positive constant $C$, there exists $\alpha=\left(\alpha_{1}, \ldots, \alpha_{n}\right)^{T}$, with $\alpha_{i}>0(i=1, \ldots, n)$ such that

$$
\min \left\{k_{i}, \quad \frac{m_{i}\left(k_{i}+1\right)}{m_{i}+1}\right\} \alpha_{i}-\sum_{j=1}^{n} m_{i j} \alpha_{j}<-C \quad(i=1, \ldots, n) .
$$

\section{Proof of Theorem}

First, we note that if $A$ is reducible, then the full system (1.1) can be reduced to several sub-systems, independent of each other. Therefore, in the following, we assume that $A$ is irreducible. In addition, we suppose that $k_{1}-m_{1} \leq k_{2}-m_{2} \leq \cdots k_{n}-m_{n}$.

Let $\varphi_{m_{i}}(x)(i=1, \ldots, n)$ be the first eigenfunction of

$$
-\Delta_{m_{i}} \varphi_{m_{i}}=\lambda \varphi_{m_{i}}^{m_{i}}(x) \quad \text { in } \quad \Omega, \quad \varphi_{m_{i}}(x)=0 \quad \text { on } \quad \partial \Omega
$$

with the first eigenvalue $\lambda_{m_{i}}$, normalized by $\left\|\varphi_{m_{i}}(x)\right\|_{\infty}=1$, then $\lambda_{m_{i}}>0, \varphi_{m_{i}}(x)>0$ in $\Omega$ and $\varphi_{m_{i}}(x) \in W_{0}^{1, m_{i}+1} \cap C^{1}(\Omega)$ and $\frac{\partial \varphi_{m_{i}}(x)}{\partial v}<0$ on $\partial \Omega$ (see [14-16]).

Thus, there exist some positive constants $A_{m_{i}}, B_{m_{i}}, C_{m_{i}}$, and $D_{m_{i}}$ such that

$$
A_{m_{i}} \leq-\frac{\partial \varphi_{m_{i}}(x)}{\partial v} \leq B_{m_{i}}, \quad\left|\nabla \varphi_{m_{i}}(x)\right| \geq C_{m_{i}}, \quad x \in \partial \Omega ; \quad\left|\nabla \varphi_{m_{i}}(x)\right| \leq D_{m_{i}}, \quad x \in \bar{\Omega}
$$


We also have $\left|\nabla \varphi_{m_{i}}(x)\right| \geq E_{m_{i}}$ provided $x \in\left\{x \in \Omega: \operatorname{dist}(x, \partial \Omega) \leq \varepsilon_{m_{i}}\right\}$ with $E_{m_{i}}=\frac{C_{m_{i}}}{2}$ and some positive constant $\varepsilon_{m_{i}}$. For the fixed $\varepsilon_{m_{i}}$, there exists a positive constant $F_{m_{i}}$ such that $\varphi_{m_{i}}(x) \geq F_{m_{i}}$ if $x \in\left\{x \in \Omega: \operatorname{dist}(x, \partial \Omega)>\varepsilon_{m_{i}}\right\}$.

Proof of the sufficiency. We divide this proof into three different cases.

Case 1. $\left(k_{i}<m_{i}(i=1, \ldots, n)\right)$. Let

$$
\bar{u}_{i}(x, t)=P_{i} e^{\alpha_{i} t} \log \left(\left(1-\varphi_{m_{i}}(x)\right) e^{\frac{\left(k_{i}-m_{i}\right) \alpha_{i} t}{m_{i}}}+Q_{i}\right) \quad(i=1, \ldots, n),
$$

where $Q_{i}$ satisfies $Q_{i} \log Q_{i} \geq \frac{2\left(m_{i}-k_{i}\right)}{m_{i}} \quad(i=1, \ldots, n)$, and constants $P_{i}, \alpha_{i}(i=1, \ldots, n)$ remain to be determined. Since $Q_{i} \log Q_{i} \geq \frac{2\left(m_{i}-k_{i}\right)}{m_{i}}$, by performing direct calculations, we have

$$
\begin{aligned}
& \left(\bar{u}_{i}^{k_{i}}\right)_{t} \geq k_{i} \alpha_{i} P_{i}^{k_{i}} e^{k_{i} \alpha_{i} t}\left(\log \left(\left(1-\varphi_{m_{i}}(x)\right) e^{\frac{\left(k_{i}-m_{i}\right) \alpha_{i} t}{m_{i}}}+Q_{i}\right)\right)^{k_{i}} \\
& +k_{i} \alpha_{i} P_{i}^{k_{i}} e^{k_{i} \alpha_{i} t}\left(\log \left(\left(1-\varphi_{m_{i}}(x)\right) e^{\frac{\left(k_{i}-m_{i}\right) \alpha_{i} t}{m_{i}}}+Q_{i}\right)\right)^{k_{i}-1} \\
& \times \frac{\frac{k_{i}-m_{i}}{m_{i}}\left(1-\varphi_{m_{i}}(x)\right) e^{\frac{\left(k_{i}-m_{i}\right) \alpha_{i} t}{m_{i}}}}{\left(1-\varphi_{m_{i}}(x)\right) e^{\frac{\left(k_{i}-m_{i}\right) \alpha_{i} t}{m_{i}}}+Q_{i}} \\
& \geq \frac{k_{i} \alpha_{i}}{2} P_{i}^{k_{i}} e^{k_{i} \alpha_{i} t}\left(\log \left(\left(1-\varphi_{m_{i}}(x)\right) e^{\frac{\left(k_{i}-m_{i}\right) \alpha_{i} t}{m_{i}}}+Q_{i}\right)\right)^{k_{i}} \\
& \geq \frac{k_{i} \alpha_{i}}{2} P_{i}^{k_{i}} e^{k_{i} \alpha_{i} t}\left(\log Q_{i}\right)^{k_{i}}, \\
& \Delta_{m_{i}} \bar{u}_{i}=\sum_{j=1}^{N}\left(\frac{P_{i}^{m_{i}} e^{k_{i} \alpha_{i} t}\left(-\left|\nabla \varphi_{m_{i}}(x)\right|^{m_{i}-1}\left(\varphi_{m_{i}}\right)_{x_{j}}\right)}{\left(\left(1-\varphi_{m_{i}}(x)\right) e^{\frac{\left(k_{i}-m_{i}\right) \alpha_{i} t}{m_{i}}}+Q_{i}\right)}\right)_{x_{j}} \leq \frac{\lambda_{m_{i}} P_{i}^{m_{i}} e^{k_{i} \alpha_{i} t}}{Q_{i}^{m_{i}}}
\end{aligned}
$$

in $\Omega \times \mathbb{R}^{+}$. By setting $c_{m_{i}}=C_{m_{i}}$ if $m_{i} \geq 1, c_{m_{i}}=D_{m_{i}}$ if $m_{i}<1$, we have one the boundary that

$$
\begin{aligned}
& \nabla_{m_{i}} \overline{u_{i}} \cdot v \geq \frac{P_{i}^{m_{i}} c_{m_{i}}^{m_{i}-1} A_{m_{i}}}{\left(1+Q_{i}\right)^{m_{i}}} e^{k_{i} \alpha_{i} t} \quad(i=1, \ldots, n), \\
& \prod_{j=1}^{n} \bar{u}_{j}^{m_{i j}} \leq \prod_{j=1}^{n}\left(P_{j} \log \left(1+Q_{j}\right)\right)^{m_{i j}} e^{\sum_{j=1}^{n} m_{i j} \alpha_{j} t} \quad(i=1, \ldots, n) .
\end{aligned}
$$

we have

$$
\nabla_{m_{i}} \bar{u}_{i} \cdot v \geq \bar{\lambda} \prod_{j=1}^{n} \bar{u}_{j}^{m_{i j}} \quad(i=1, \ldots, n)
$$

if

$$
\frac{P_{i}^{m_{i}} c_{m_{i}}^{m_{i}-1} A_{m_{i}}}{\left(1+Q_{i}\right)^{m_{i}}} \geq \bar{\lambda} \prod_{j=1}^{n}\left(P_{j} \log \left(1+Q_{j}\right)\right)^{m_{i j}} \quad(i=1, \ldots, n)
$$


and

$$
k_{i} \alpha_{i} \geq \sum_{j=1}^{n} m_{i j} \alpha_{j}(i=1, \ldots, n)
$$

Note that $k_{i}<m_{i}(i=1, \ldots, n)$. From Lemmas 2.2 and 2.3, we know that inequalities (3.4) and (3.5) hold for suitable choices of $P_{i}, \alpha_{i}(i=1, \ldots, n)$. Moreover, if we choose $P_{i}, \alpha_{i}$ to be large enough such that

$$
P_{i} \log Q_{i} \geq\left\|u_{i 0}\right\|_{\infty}, \quad \alpha_{i} \geq \frac{2 \lambda_{m_{i}} P_{i}^{m_{i}-k_{i}}}{k_{i} Q_{i}^{m_{i}}\left(\log Q_{i}\right)^{k_{i}}}
$$

then $\overline{u_{i}}(x, 0) \geq u_{i 0},\left(\bar{u}_{i}^{k_{i}}\right)_{t} \geq \Delta_{m_{i}} \bar{u}_{i} \quad(i=1, \ldots, n)$. Therefore, we have proved that $\left(\bar{u}_{1}, \ldots, \bar{u}_{n}\right)$ is a global upper solution of the system (1.1). The global existence of solutions to the problem (1.1) follows from the comparison principle.

Case 2. $\left(k_{i} \geq m_{i}(i=1, \ldots, n)\right)$. Let

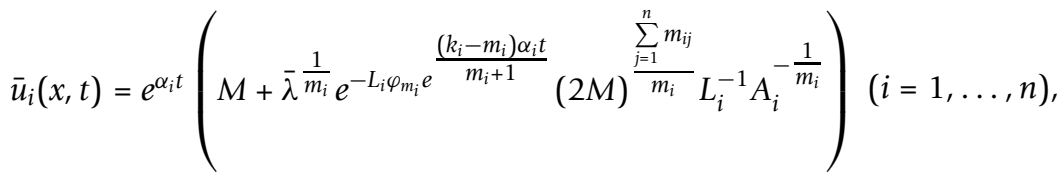

where $A_{i}=A_{m_{i}} C_{m_{i}}^{m_{i}-1}$ if $m_{i} \geq 1, A_{i}=A_{m_{i}} D_{m_{i}}^{m_{i}-1}$ if $m_{i}<1, \varphi_{m_{i}}, A_{m_{i}}, B_{m_{i}}, C_{m_{i}}$ are defined in (3.1) and (3.2), $\alpha_{i}(i=1, \ldots, n)$ are positive constants that remain to be determined, and

$$
M=\max _{1 \leq i \leq n}\left\{1,\left\|u_{i 0}\right\|_{\infty}\right\}, \quad L_{i}=\bar{\lambda}^{\frac{1}{m_{i}}} 2^{\frac{\sum_{j=1}^{n} m_{i j}}{m_{i}}} M^{\frac{\sum_{j=1}^{n} m_{i j}-m_{i}}{m_{i}}} A_{i}^{-\frac{1}{m_{i}}} \max \left\{1, \quad \frac{2\left(k_{i}-m_{i}\right)}{m_{i}+1}\right\} .
$$

Since $-y e^{-y} \geq-e^{-1}$ for any $y>0$, we know that

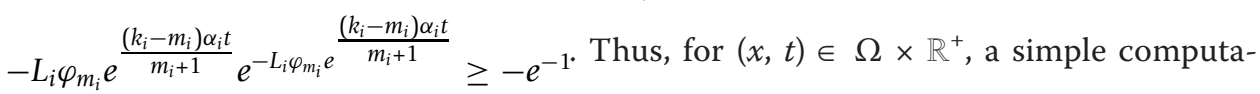
tion shows that

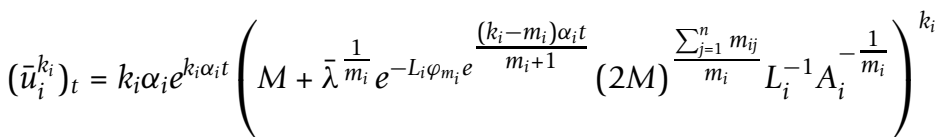

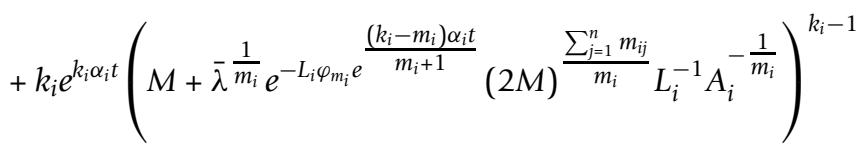

$$
\begin{aligned}
& \times \bar{\lambda}^{\frac{1}{m_{i}}}(2 M)^{\frac{\sum_{j=1}^{n} m_{i j}}{m_{i}}} L_{i}^{-1} A_{i}^{-\frac{1}{m_{i}}} \frac{\left(k_{i}-m_{i}\right) \alpha_{i}}{m_{i}+1}\left(-L_{i} \varphi_{m_{i}}\right) e^{\frac{\left(k_{i}-m_{i}\right) \alpha_{i} t}{m_{i}+1}} e^{-L_{i} \varphi_{m_{i}} e^{\frac{\left(k_{i}-m_{i}\right) \alpha_{i} t}{m_{i}+1}}} \\
& \geq \frac{1}{2} k_{i} \alpha_{i} e^{k_{i} \alpha_{i} t} .
\end{aligned}
$$

In addition, we have

$$
\begin{aligned}
\Delta_{m_{i}} \bar{u}_{i} \leq & \bar{\lambda} \lambda_{m_{i}}(2 M)^{\sum_{j=1}^{n} m_{i j}} A_{i}^{-1} \varphi_{m_{i}}^{m_{i}} e^{m_{i} \alpha_{i} t} e^{\frac{m_{i}\left(k_{i}-m_{i}\right) \alpha_{i} t}{m_{i}+1}} e^{-L_{i} m_{i} \varphi_{m_{i}} e^{\frac{\left(k_{i}-m_{i}\right) \alpha_{i} t}{m_{i}+1}}} \\
& +\bar{\lambda} L_{i} m_{i}(2 M)^{\sum_{j=1}^{n} m_{i j}} A_{i}^{-1} e^{k_{i} \alpha_{i} t} e^{-L_{i} m_{i} \varphi_{m_{i}} e^{\frac{\left(k_{i}-m_{i}\right) \alpha_{i} t}{m_{i}+1}}\left|\nabla \varphi_{m_{i}}\right|^{m_{i}+1}} \\
\leq & \bar{\lambda}\left(\lambda_{m_{i}}+L_{i} m_{i} D_{m_{i}}^{m_{i}+1}\right)(2 M)^{\sum_{j=1}^{n} m_{i j}} A_{i}^{-1} e^{k_{i} \alpha_{i} t} .
\end{aligned}
$$


Noting $\varphi_{m_{i}}=0(i=1,2, \ldots, n)$ on $\partial \Omega$, we have on the boundary that

$$
\begin{gathered}
\nabla_{m_{i}} \bar{u}_{i} \cdot v \geq \bar{\lambda}(2 M)^{\sum_{j=1}^{n} m_{i j}} e^{\frac{m_{i}\left(k_{i}-m_{i}\right) \alpha_{i} t}{m_{i}+1}}, \\
\prod_{j=1}^{n} \bar{u}_{j}^{m_{i j}} \leq(2 M)^{\sum_{j=1}^{n} m_{i j}} e^{\sum_{j=1}^{n} m_{i j} \alpha_{j} t} .
\end{gathered}
$$

Then, we have

$$
\nabla_{m_{i}} \bar{u}_{i} \cdot v \geq \bar{\lambda} \prod_{j=1}^{n} \bar{u}_{j}^{m_{i j}}(i=1, \ldots, n)
$$

if

$$
\frac{m_{i}\left(k_{i}-1\right) \alpha_{i}}{m_{i}+1} \geq \sum_{j=1}^{n} m_{i j} \alpha_{j}(i=1, \ldots, n)
$$

From Lemma 2.2, we know that inequalities (3.7) hold for suitable choices of $\alpha_{i}(i=$ $1, \ldots, n)$. Moreover, if we choose $\infty_{i}$ to be large enough such that

$$
\alpha_{i} \geq 2 \bar{\lambda}\left(\lambda_{m_{i}}+L_{i} m_{i} D_{m_{i}}^{m_{i}+1}\right)(2 M)^{\sum_{j=1}^{n} m_{i j}}\left(k_{i} A_{i}\right)^{-1},
$$

then $\left(\bar{u}_{i}^{k_{i}}\right)_{t} \geq \Delta_{m_{i}} \bar{u}_{i}(i=1, \ldots, n)$. Therefore, we have shown that $\left(\bar{u}_{1}, \ldots, \bar{u}_{n}\right)$ is an upper solution of $(1.1)$ and exists globally. Therefore, $\left(u_{1}, \ldots, u_{n}\right) \leq\left(\bar{u}_{1}, \ldots, \bar{u}_{n}\right)$, and hence the solution $\left(u_{1}, \ldots, u_{n}\right)$ of $(1.1)$ exists globally.

Case 3. $\left(k_{i}<m_{i}(i=1, \ldots, s) ; k_{i} \geq m_{i}(i=s+1, \ldots, n)\right)$. Let $\bar{u}_{i}(x, t)(i=1, \ldots, s)$ be as in (3.3) and

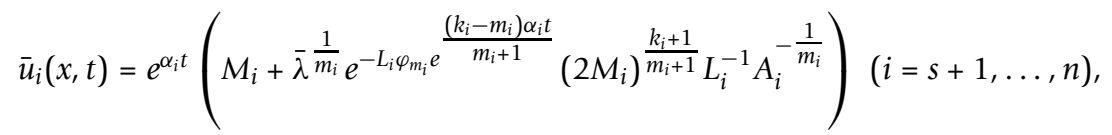

where $\varphi_{m_{i}}$, and $A_{i}$ are as in case 2. By Lemma 2.3, we choose $P_{i} \geq\left(\log Q_{i}\right)^{-1}|| u_{i 0} \|_{\infty}(i$ $=1, \ldots, s)$ and $M_{i} \geq \max \left\{1,\left\|u_{i 0}\right\|_{\infty}\right\}(i=s+1, \ldots, n)$ such that

$$
\begin{aligned}
& \frac{P_{i}^{m_{i}} c_{m_{i}}^{m_{i}-1} A_{m_{i}}}{\left(1+Q_{i}\right)^{m_{i}}} \geq \prod_{j=1}^{s}\left(P_{j} \log \left(1+Q_{j}\right)\right)^{m_{i j}} \prod_{j=s+1}^{n}\left(2 M_{j}\right)^{m_{i j}} \quad(i=1, \ldots, s), \\
& \bar{\lambda}\left(2 M_{i}\right)^{\frac{m_{i}\left(k_{i}+1\right)}{m_{i}+1}} \geq \prod_{j=1}^{s}\left(P_{j} \log \left(1+Q_{j}\right)\right)^{m_{i j}} \prod_{j=s+1}^{n}\left(2 M_{j}\right)^{m_{i j}} \quad(i=s+1, \ldots, n) .
\end{aligned}
$$

Set

$$
L_{i}=\bar{\lambda}^{\frac{1}{m_{i}}} 2^{\frac{k_{i}+1}{m_{i}+1}} M_{i}^{\frac{k_{i}-m_{i}}{m_{i}+1}} A_{i}^{-\frac{1}{m_{i}}} \max \left\{1, \frac{2\left(k_{i}-m_{i}\right)}{m_{i}+1}\right\} \quad(i=s+1, \ldots, n) .
$$


By similar arguments, in cases 1 and 2, we have on the boundary that

$$
\begin{aligned}
& \nabla_{m_{i}} \bar{u}_{i} \cdot v \geq \frac{P_{i}^{m_{i}} c_{m_{i}}^{m_{i}-1} A_{m_{i}}}{\left(1+Q_{i}\right)^{m_{i}}} e^{k_{i} \alpha_{i} t} \quad(i=1, \ldots, s), \\
& \nabla_{m_{i}} \bar{u}_{i} \cdot v \geq \bar{\lambda}\left(2 M_{i}\right)^{\frac{m_{i}\left(k_{i}+1\right)}{m_{i}+1}} e^{\frac{m_{i}\left(k_{i}-1\right) \alpha_{i} t}{m_{i}+1}} \quad(i=s+1, \ldots, n), \\
& \prod_{j=1}^{n} \bar{u}_{j}^{m_{i j}} \leq \prod_{j=1}^{s}\left(P_{j} \log \left(1+Q_{j}\right)\right)^{m_{i j}} \prod_{j=s+1}^{n}\left(2 M_{j}\right)^{m_{i j}} e^{\sum_{j=1}^{n} m_{i j} \alpha_{j} t} \quad(i=1, \ldots, n) .
\end{aligned}
$$

Therefore employing (3.8), we see that

$$
\nabla_{m_{i}} \bar{u}_{i} \cdot v \geq \bar{\lambda} \prod_{j=1}^{n} \bar{u}_{j}^{m_{i j}}(i=1, \ldots, n)
$$

if we knew

$$
k_{i} \alpha_{i} \geq \sum_{j=1}^{n} m_{i j} \alpha_{j}(i=1, \ldots, s), \quad \frac{m_{i}\left(k_{i}-1\right) \alpha_{i}}{m_{i}+1} \geq \sum_{j=1}^{n} m_{i j} \alpha_{j}(i=s+1, \ldots, n) .
$$

We deduce from Lemma 2.2 that (3.9) holds for suitable choices of $\alpha_{i}(i=1, \ldots, n)$. Moreover, we can choose $\alpha_{i}$ large enough to assure that

$$
\begin{aligned}
& \alpha_{i} \geq \frac{2 \lambda_{m_{i}} P_{i}^{m_{i}-k_{i}}}{k_{i} Q_{i}^{i_{i}}\left(\log Q_{i}\right)^{k_{i}}},(i=1, \ldots, s), \\
& \alpha_{i} \geq 2 \bar{\lambda}\left(\lambda_{m_{i}}+L_{i} m_{i} D_{m_{i}}^{m_{i}+1}\right)\left(2 M_{i}\right)^{\frac{m_{i}\left(k_{i}+1\right)}{m_{i}+1}}\left(k_{i} A_{i}\right)^{-1}(i=s+1, \ldots, n),
\end{aligned}
$$

Then, as in the calculations of cases 1 and 2 , we have $\left(\bar{u}_{i}^{k_{i}}\right)_{t} \geq \Delta_{m_{i}} \bar{u}_{i} \quad(i=1, \ldots, n)$. We prove that $\left(\bar{u}_{1}, \ldots, \bar{u}_{n}\right)$ is an upper solution of $(1.1)$, so $\left(u_{1}, \ldots, u_{n}\right)$ exists globally.

\section{Proof of the necessity.}

Without loss of generality, we first assume that all the lower-order principal minor determinants of $\mathrm{A}$ are non-negative, and $|A|<0$, for, if not, there exists some $l$ thorder $(1 \leq l<n)$ principal minor determinant $\operatorname{det} A_{l \times l}$ of $A=\left(a_{i j}\right)_{n \times n}$ which is negative. Without loss of generality, we may consider that

$$
A_{l \times l}=\left(\begin{array}{ccc}
a_{11} & \ldots & a_{1 l} \\
a_{12} & \ldots & a_{2 l} \\
\ldots & \ldots & \ldots \\
a_{l 1} & \ldots & a_{l l}
\end{array}\right)
$$

and all of the sth-order $(1 \leq s \leq l-1)$ principal minor determinants $\operatorname{det} A_{s \times s}$ of $A_{l \times l}$ are non-negative. Then, we consider the following problem:

$$
\begin{array}{ll}
\left(w_{i}^{k_{i}}\right)_{t}=\Delta_{m_{i}} w_{i}(i=1, \ldots, l), & x \in \Omega, t>0, \\
\nabla_{m_{i}} w_{i} \cdot v=\delta^{\sum_{j=l+1}^{n} m_{i j}} \prod_{j=1}^{n} w_{j}^{m_{i j}}(i=1, \ldots, l), & x \in \partial \Omega, t>0, \\
w_{i}(x, 0)=u_{i 0}(x)(i=1, \ldots, l), & x \in \bar{\Omega} .
\end{array}
$$

Note that $\delta=\min _{1 \leq i \leq n}\left\{\min _{\bar{\Omega}} u_{i 0}(x)\right\}>0$. If we can prove that the solution $\left(w_{1}, \ldots, w_{l}\right)$ of (3.10) blows up in finite time, then $\left(w_{1}, \ldots w_{l}, \delta, \ldots, \delta\right)$ is a lower solution of (1.1) that blows up in finite time. Therefore, the solution of (1.1) blows up in finite time.

We will complete the proof of the necessity of our theorem in three different cases. 
Case 1. $\left(k_{i}<m_{i}(i=1, \ldots, n)\right)$. Let

$$
\underline{u}_{i}=Y_{i}^{\rho_{i}} \quad \text { and } \quad Y_{i}=a h^{1+\frac{1}{m_{i}}}(x)+(b-c t)^{-\gamma_{i}}(i=1, \ldots, n),
$$

where $h(x)=\sum_{i=1}^{N} x_{i}+N d+1, d=\max \{|x| \mid x \in \bar{\Omega}\}, \quad \rho_{i}=\frac{m_{i}+\frac{1}{\gamma_{i}}}{m_{i}-k_{i}}, \gamma_{i}=\frac{\left(m_{i}-k_{i}\right) \alpha_{i}-1}{m_{i}}$, the $\alpha_{i}$ are as given in Lemma 2.4 and satisfy $\alpha_{i}>\frac{1}{m_{i}-k_{i}}$,

$$
\begin{aligned}
& b=\max _{1 \leq i \leq n}\left\{1,\left(\frac{1}{2} \delta^{\frac{1}{\rho_{i}}}\right)^{-\frac{1}{\gamma_{i}}}\right\}, a=\min _{1 \leq i \leq n}\left\{b^{-\gamma_{i}}(2 N d+1)^{-\frac{1+m_{i}}{m_{i}}},\right. \\
& \left.\left(\underline{\lambda}^{-1}\left[\frac{\left(1+m_{i}\right) \rho_{i} N^{\frac{1}{2}} 2^{\rho_{i}-1}}{m_{i}}\right](2 N d+1)\right)^{-\frac{1}{m_{i}}} b^{-\frac{\sum_{j=1}^{n} m_{i j} \alpha_{j}}{m_{i}}}\right\} \\
& c=\min _{1 \leq i \leq n}\left\{\frac{a^{m_{i}} \rho_{i}^{m_{i}-1}\left(1+\frac{1}{m_{i}}\right)^{m_{i}} N^{\frac{m_{i}+1}{2}}}{k_{i} \gamma_{i}}\right\} .
\end{aligned}
$$

By direct computation for $(x, t) \in \Omega \times\left(0, \frac{b}{c}\right)$, we have

$$
\begin{gathered}
\left(\underline{u}_{i}^{k_{i}}\right)_{t}=c k_{i} \rho_{i} \gamma_{i} Y_{i}^{k_{i} \rho_{i}-1}(b-c t)^{-\left(\gamma_{i}+1\right)}, \quad \nabla \underline{u}_{i}=a \rho_{i}\left(1+\frac{1}{m_{i}}\right) Y_{i}^{\rho_{i}-1} h^{\frac{1}{m_{i}}}(x)(1, \ldots, 1), \\
\Delta_{m_{i}} \underline{u}_{i}=\sum_{j=1}^{N}\left(\left(a \rho_{i}\left(1+\frac{1}{m_{i}}\right)\right)^{m_{i}} N^{\frac{m_{i}-1}{2}} Y_{i}^{m_{i}\left(\rho_{i}-1\right)} h(x)\right)_{x_{j}} \\
=\left(a \rho_{i}\left(1+\frac{1}{m_{i}}\right)\right)^{m_{i}} N^{\frac{m_{i}+1}{2}} Y_{i}^{m_{i}\left(\rho_{i}-1\right)} \\
+m_{i}\left(\rho_{i}-1\right) \rho_{i}^{m_{i}}\left(a\left(1+\frac{1}{m_{i}}\right)\right)^{m_{i}+1} N^{\frac{m_{i}+1}{2}} h^{1+\frac{1}{m_{i}}}(x) Y_{i}^{m_{i}\left(\rho_{i}-1\right)-1} \\
\geq\left(a \rho_{i}\left(1+\frac{1}{m_{i}}\right)\right)^{m_{i}} N^{\frac{m_{i}+1}{2}} Y_{i}^{k_{i} \rho_{i}-1} Y_{i}^{m_{i}\left(\rho_{i}-1\right)-k_{i} \rho_{i}+1} \\
\geq\left(\underline{u}_{i}^{k_{i}}\right)_{t}(i=1, \ldots, n) .
\end{gathered}
$$

For $(x, t) \in \partial \Omega \times\left(0, \frac{b}{c}\right)$, we have

$$
\begin{aligned}
\nabla_{m_{i}} \underline{u}_{i} \cdot v & \leq\left(a \rho_{i}\left(1+\frac{1}{m_{i}}\right)\right)^{m_{i}} N^{\frac{m_{i}}{2}}(2 N d+1) 2^{m_{i}\left(\rho_{i}-1\right)}(b-c t)^{-m_{i}\left(\rho_{i}-1\right) \gamma_{i}} \\
& =\left(a \rho_{i}\left(1+\frac{1}{m_{i}}\right)\right)^{m_{i}} N^{\frac{m_{i}}{2}}(2 N d+1) 2^{m_{i}\left(\rho_{i}-1\right)}(b-c t)^{-\left(k_{i} \alpha_{i}+1\right)}(i=1, \ldots, n), \\
\prod_{j=1}^{n} \underline{u}_{j}^{m_{i j}} & =\prod_{j=1}^{n} Y_{j}^{m_{i j} \rho_{j}} \geq \prod_{j=1}^{n}(b-c t)^{-\sum_{j=1}^{n} m_{i j} \alpha_{j}}(i=1, \ldots, n) .
\end{aligned}
$$

Thus, by (3.12) and Lemma 2.4, we have

$$
\nabla_{m_{i}} \underline{u}_{i} \cdot v \leq \underline{\lambda} \prod_{j=1}^{n} \underline{u}_{j}^{m_{i j}}(i=1, \ldots, n) .
$$

We confirm that $\left(\underline{u}_{1}, \ldots, \underline{u}_{n}\right)$ is a lower solution of (1.1), which blows up in finite time. We know by the comparison principle that the solution $\left(u_{1}, \ldots, u_{n}\right)$ blows up in finite time.

Case 2. $\left(k_{i} \geq m_{i}(i=1, \ldots, n)\right)$. Let $d_{m_{i}}=C_{m_{i}}$ if $m_{i}<1, d_{m_{i}}=D_{m_{i}}$ if $m_{i} \geq 1$. for $k_{i} \geq m_{i}(i$ $=1, \ldots, n)$, set 


$$
\underline{u}_{i}=\frac{1}{(b-c t)^{\alpha_{i}}} e^{\frac{-a \varphi_{m_{i}}(x)}{(b-c t)^{\beta_{i}}}}
$$

where $\alpha_{i}(i=1, \ldots, n)$ are to determined later and

$$
\begin{aligned}
& \beta_{i}=\frac{\left(k_{i}-m_{i}\right) \alpha_{i}+1}{m_{i}+1}, b=\max _{1 \leq i \leq n}\left\{1, \delta^{-\frac{1}{\alpha_{i}}}\right\}, \\
& a=\min _{1 \leq i \leq n}\left\{1, \underline{\lambda}^{\frac{1}{m_{i}}}\left(B_{m_{i}} d_{m_{i}}^{m_{i}-1}\right)^{-\frac{1}{m_{i}}} b \frac{-\sum_{j=1}^{n} m_{i j} \alpha_{j}}{m_{i}}\right\}, \\
& c=\min _{1 \leq i \leq n}\left\{\frac{m_{i} a^{m_{i}+1} E_{m_{i}}^{m_{i}+1}}{k_{i} \alpha_{i}}, \frac{\lambda_{m_{i}}\left(k_{i}-m_{i}\right) a^{m_{i}+1} F_{m_{i}}^{m_{i}+1}}{k_{i} \alpha_{i}}\right\} .
\end{aligned}
$$

By a direct computation, for $x \in \Omega, 0<t<c / b$, we obtain that

$$
\begin{aligned}
& \left(\underline{u}_{i}^{k_{i}}\right)_{t}=k_{i} \alpha_{i} c e^{\frac{-a k_{i} \varphi_{m_{i}}(x)}{(b-c t)^{\beta_{i}}}}(b-c t)^{-\left(k_{i} \alpha_{i}+1\right)}-\frac{e^{\frac{-a k_{i} \varphi_{m_{i}}(x)}{(b-c t)^{\beta_{i}}}} a k_{i} \beta_{i} c \varphi_{m_{i}}(x)}{(b-c t)^{k_{i} \alpha_{i}}(b-c t)^{\beta_{i}+1}} \\
& \leq k_{i} \alpha_{i} c e^{\frac{-a k_{i} \varphi_{m_{i}}(x)}{(b-c t)^{\beta_{i}}}}(b-c t)^{-\left(k_{i} \alpha_{i}+1\right)}, \\
& \Delta_{m_{i}} \underline{u}_{i}=\frac{\lambda_{m_{i}} a^{m_{i}} \varphi_{m_{i}}^{m_{i}} e^{\frac{-a m_{i} \varphi_{m_{i}}(x)}{(b-c t)^{\beta_{i}}}}}{(b-c t)^{m_{i}\left(\alpha_{i}+\beta_{i}\right)}}+\frac{m_{i} a^{m_{i}+1} e^{\frac{-a m_{i} \varphi_{m_{i}}(x)}{(b-c t)^{\beta_{i}}}}\left|\nabla \varphi_{m_{i}}\right|^{m_{i}+1}}{(b-c t)^{m_{i}\left(\alpha_{i}+\beta_{i}\right)+\beta_{i}}} .
\end{aligned}
$$

If $x \in\left\{x \in \Omega: \operatorname{dist}(x, \partial \Omega)>\varepsilon_{m_{i}}\right\}$, we have $\varphi_{m_{i}} \geq F_{m_{i}}$, and thus

$$
\Delta_{m_{i}} \underline{u}_{i} \geq \frac{\lambda_{m_{i}} a^{m_{i}} F_{m_{i}}^{m_{i}} e^{\frac{-a_{i} m_{i} \varphi_{m_{i}}(x)}{(b-c t)^{\beta_{i}}}}}{(b-c t)^{m_{i}\left(\alpha_{i}+\beta_{i}\right)}} .
$$

On the other hand, since $-y e^{-y} \geq-e^{-1}$ for any $y>0$, we have

$$
\left(\underline{u}_{i}^{k_{i}}\right)_{t} \leq k_{i} \alpha_{i} c e^{\frac{-a k_{i} \varphi_{m_{i}}(x)}{(b-c t)^{\beta_{i}}}}(b-c t)^{-\left(k_{i} \alpha_{i}+1\right)} \leq \frac{k_{i} \alpha_{i} c e^{\frac{-a m_{i} \varphi_{m_{i}}(x)}{(b-c t)^{\beta_{i}}}}}{a\left(k_{i}-m_{i}\right) F_{m_{i}} e(b-c t)^{m_{i}\left(\alpha_{i}+\beta_{i}\right)}} .
$$

We have by (3.16), (3.18), and (3.19) that $\left(\underline{u}_{i}^{k_{i}}\right)_{t} \leq \Delta_{m_{i}} \underline{u}_{i}(i=1, \ldots, n)$. If $x \in\left\{x \in \Omega: \operatorname{dist}(x, \partial \Omega) \leq \varepsilon_{m_{i}}\right\}$, then $\left|\nabla \varphi_{m_{i}}\right| \geq E_{m_{i}}$, and then

$$
\Delta_{m_{i}} \underline{u}_{i} \geq \frac{m_{i} a^{m_{i}+1} E_{m_{i}}^{m_{i}+1} e^{\frac{-a k_{i} \varphi_{m_{i}}(x)}{(b-c t)^{\beta_{i}}}}}{(b-c t)^{m_{i}\left(\alpha_{i}+\beta_{i}\right)+\beta_{i}}}=\frac{m_{i} a^{m_{i}+1} E_{m_{i}}^{m_{i}+1} e^{\frac{-a k_{i} \varphi_{m_{i}}(x)}{(b-c t)^{\beta_{i}}}}}{(b-c t)^{k_{i} \alpha_{i}+1}} .
$$

It follows from (3.16), (3.17), and (3.20) that $\left(\underline{u}_{i}^{k_{i}}\right)_{t} \leq \Delta_{m_{i}} \underline{u}_{i}(i=1, \ldots, n)$.

We have on the boundary that

$$
\begin{aligned}
\nabla_{m_{i}} \underline{u}_{i} \cdot v & =\frac{a^{m_{i}}\left|\nabla \varphi_{m_{i}}\right|^{m_{i}-1} e^{\frac{-a m_{i} \varphi_{m_{i}}(x)}{(b-c t)^{\beta_{i}}}}\left(-\frac{\partial \varphi_{m_{i}}}{\partial v}\right)}{(b-c t)^{m_{i}\left(\alpha_{i}+\beta_{i}\right)}} \leq \frac{a^{m_{i}} B_{m_{i}} d_{m_{i}}^{m_{i}-1}}{(b-c t)^{m_{i}\left(\alpha_{i}+\beta_{i}\right)}}(i=1, \ldots, n), \\
\prod_{j=1}^{n} \underline{u}_{j}{ }^{m_{i j}} & =\frac{1}{(b-c t)^{\sum_{j=1}^{n} m_{i j} \alpha_{j}}}(i=1,2, \ldots, n) .
\end{aligned}
$$


Moreover, by (3.14) and Lemma 2.4, we have that

$$
m_{i}\left(\alpha_{i}+\beta_{i}\right) \leq \sum_{j=1}^{n} m_{i j} \alpha_{j}(i=1, \ldots, n) .
$$

(3.15), (3.21), and (3.22) imply that $\nabla_{m_{i}} \underline{u}_{i} \cdot v \leq \underline{\lambda} \prod_{j=1}^{n} \underline{u}_{j}^{m_{i j}}(i=1, \ldots, n)$. Therefore, $\left(\underline{u}_{1}, \ldots, \underline{u}_{1}\right)$ is a lower solution of $(1.1)$.

For $k_{i}=m_{i}(i=1, \ldots, n)$, let

$$
\underline{u}_{i}=\frac{1}{(b-c t)^{\alpha_{i}}} e^{\frac{-a \varphi_{m_{i}}(x)}{(b-c t)^{\frac{1}{m_{i}}}}} .
$$

For $k_{i}=m_{i}(i=1, \ldots, s)$ and $k_{i}>m_{i}(i=s+1, \ldots, n)$, let $\bar{u}_{i}(x, t)$ as in (3.13) and (3.23). Using similar arguments as above, we can prove that $\left(\underline{u}_{1}, \ldots, \underline{u}_{n}\right)$ is a lower solution of (1.1). Therefore, $\left(\underline{u}_{1}, \ldots, \underline{u}_{n}\right) \leq\left(u_{1}, \ldots, u_{n}\right)$. Consequently, $\left(u_{1}, \ldots, u_{n}\right)$ blows up in finite time.

Case 3. $\left(k_{i}<m_{i}(i=1, \ldots, s) ; k_{i} \geq m_{i}(i=s+1, \ldots, n)\right)$. Let $\bar{u}_{i}(x, t)(i=1, \ldots, s)$ be as in (3.11) and

$$
\underline{u}_{i}=\frac{1}{(b-c t)^{\alpha_{i}}} e^{\frac{-a \varphi_{m_{i}}(x)}{(b-c t)^{\beta_{i}}}}(i=s+1, \ldots, n),
$$

where $\alpha_{i}^{\prime}$ s are to determined later and

$$
\begin{aligned}
& \beta_{i}=\frac{\left(k_{i}-m_{i}\right) \alpha_{i}+1}{m_{i}+1}(i=s+1, \ldots, n), \quad b=\max \left\{1, \max _{1 \leqslant i \leqslant s}\left\{\left(\frac{1}{2} \delta^{\frac{1}{\rho_{i}}}\right)^{-\frac{1}{\gamma_{i}}}\right\}, \quad \max _{s+1 \leqslant i \leqslant n}\left\{\delta^{-\frac{1}{\alpha_{i}}}\right\}\right\}, \\
& a=\min \left\{\min _{s+1 \leqslant i \leqslant n}\left\{\underline{\lambda} \frac{1}{m_{i}}\left(B_{m_{i}} d_{m_{i}}^{m_{i}-1}\right)^{-\frac{1}{m_{i}}} b^{\frac{-\sum_{j=1}^{n} m_{i j} \alpha_{j}}{m_{i}}}\right\}, \min _{1 \leqslant i \leqslant s}\left\{b^{-\gamma_{i}}(2 N d+1)^{-\frac{1+m_{i}}{m_{i}}},\right.\right. \\
& \left.\left.\left(\underline{\lambda}^{-1}\left[\frac{\left(1+m_{i}\right) \rho_{i} N^{\frac{1}{2}} 2^{\rho_{i}-1}}{m_{i}}\right](2 N d+1)\right)^{-\frac{1}{m_{i}}} b^{-\frac{-\sum_{j=1}^{n} m_{i j} \alpha_{j}}{m_{i}}}\right\}\right\} \\
& c=\min \left\{\min _{1 \leqslant i \leqslant s}\left\{\frac{a^{m_{i}} \rho_{i}^{m_{i}-1}\left(1+\frac{1}{m_{i}}\right)^{m_{i}} N \frac{m_{i}+1}{2}}{k_{i} \gamma_{i}}\right\},\right. \\
& \left.\min _{s+1 \leqslant i \leqslant n}\left\{\frac{m_{i} a^{m_{i}+1} E_{m_{i}}^{m_{i}+1}}{k_{i} \alpha_{i}}, \frac{\lambda_{m_{i}}\left(k_{i}-m_{i}\right) a^{m_{i}+1} F_{m_{i}}^{m_{i}+1}}{k_{i} \alpha_{i}}\right\}\right\}
\end{aligned}
$$

Based on arguments in cases 1 and 2 , we have $\left(\underline{u}_{i}^{k_{i}}\right)_{t} \leq \Delta_{m_{i}} \underline{u}_{i}(i=1, \ldots, n)$ for $(x, t) \in \Omega \times\left(0, \frac{b}{c}\right)$. Furthermore, for $(x, t) \in \partial \Omega \times\left(0, \frac{b}{c}\right)$, we have

$$
\begin{aligned}
& \nabla_{m_{i}} \underline{u}_{i} \cdot v \leq\left(a \rho_{i}\left(1+\frac{1}{m_{i}}\right)\right)^{m_{i}} N^{\frac{m_{i}}{2}}(2 N d+1) 2^{m_{i}\left(\rho_{i}-1\right)}(b-c t)^{-\left(k_{i} \alpha_{i}+1\right)}(i=1, \ldots, s), \\
& \nabla_{m_{i}} \underline{u}_{i} \cdot v \leq a^{m_{i}} B_{m_{i}} d_{m_{i}}^{m_{i}-1}(b-c t)^{-m_{i}\left(\alpha_{i}+\beta_{i}\right)}(i=s+1, \ldots, n), \\
& \prod_{j=1}^{n} \underline{u}_{j}^{m_{i j}} \geq(b-c t)^{-\sum_{j=1}^{n} m_{i j} \alpha_{j}}(i=1, \ldots, n) .
\end{aligned}
$$

Thus,

$$
\nabla_{m_{i}} \underline{u}_{i} \cdot v \leq \underline{\lambda} \prod_{j=1}^{n} \underline{u}_{j}^{m_{i j}}(i=1, \ldots, n)
$$


holds if

$$
\begin{aligned}
k_{i} \alpha_{i}+1 & \leq \sum_{j=1}^{n} m_{i j} \alpha_{j} \quad(i=1, \ldots, s), \\
m_{i}\left(\alpha_{i}+\beta_{i}\right) & \leq \sum_{j=1}^{n} m_{i j} \alpha_{j} \quad(i=s+1, \ldots, n) .
\end{aligned}
$$

From Lemma 2.4, we know that inequalities (3.24) hold for suitable choices of $\alpha_{i}$ ( $i=$ $1, \ldots, n)$. We show that $\left(\underline{u}_{1}, \ldots, \underline{u}_{n}\right)$ is a lower solution of $(1.1)$. Since $\left(\underline{u}_{1}, \ldots, \underline{u}_{n}\right)$ blows up in finite time, it follows that the solution of (1.1) blows up in finite time.

\section{Acknowledgements}

This study was partially supported by the Projects Supported by Scientific Research Fund of SiChuan Provincial Education Department(09ZC011), and partially supported by the Natural Science Foundation Project of China West Normal University (07B046).

\section{Authors' contributions}

DW carried out all studies in the paper. LZ participated in the design of the study in the paper.

\section{Competing interests}

The authors declare that they have no competing interests.

Received: 9 November 2010 Accepted: 21 June 2011 Published: 21 June 2011

\section{References}

1. Kalashnikov, AS: Some problems of the qualitative theory of nonlinear degenerate parabolic equations of second order. Russ Math Surv. 42, 169-222 (1987)

2. $\mathrm{Li}, \mathrm{ZP}, \mathrm{Mu}, \mathrm{CL}$ : Critical exponents for a fast diffusive polytropic filtration equation with nonlinear boundary condition. J Math Anal Appl. 346, 55-64 (2008). doi:10.1016/j.jmaa.2008.05.035

3. Vazquez, JL: The Porous Medium Equations: Mathematical Theory. Oxford Mathematical Monographs. Oxford University Press, Oxford (2007)

4. Wu, ZQ, Zhao, JN, Yin, JX, Li, HL: Nonlinear Diffusion Equations. Word Scientific Publishing Co. Inc., River Edge, NJ (2001)

5. $\mathrm{Li}, \mathrm{ZP}, \mathrm{Mu}, \mathrm{CL}, \mathrm{Li}, \mathrm{YH}$ : Blowup for a non-Newtonian polytropic filtration system coupled via nonlinear boundary flux. Boundary Value Problems 2008 (2008). Article ID 847145

6. Qi, YW, Wang, MX, Wang, ZJ: Existence and non-existence of global solutions of diffusion systems with nonlinear boundary conditions. Proc R Soc Edinb A. 134, 1199-1217 (2004). doi:10.1017/S030821050000370X

7. Sun, WJ, Wang, S: Nonlinear degenerate parabolic equation with nonlinear boundary condition. Acta Mathematica Sinica, English Series. 21, 847-854 (2005). doi:10.1007/s10114-004-0512-2

8. Wang, MX: Long time behaviors of solutions of quasilinear equations with nonlinear boundary conditions. Acta Math Sinica (in Chinese) 39, 118-124 (1996)

9. Wang, MX, Wang, YM: Reaction diffusion systems with nonlinear boundary conditions. Sci China A. 27, 834-840 (1996)

10. Wang, MX, Wang, S: Quasilinear reaction-diffusion systems with nonlinear boundary conditions. J Math Anal Appl. 231, 21-33 (1999). doi:10.1006/jmaa.1998.6220

11. Wang, MX: Fast-slow diffusion systems with nonlinear boundary conditions. Nonlinear Anal. 46, 893-908 (2001). doi:10.1016/S0362-546X(00)00156-5

12. Wang, S, Wang, MX, Xie, CH: Quasilinear parabolic systems with nonlinear boundary conditions. J Differential Equations. 166, 251-265 (2000). doi:10.1006/jdeq.2000.3784

13. Wang, S: Doubly nonlinear degenerate parabolic systems with coupled nonlinear boundary conditions. J Differential Equations. 182, 431-469 (2002). doi:10.1006/jdeq.2001.4101

14. Lindqvist, P: On the equation $\operatorname{div}\left(|\nabla u|^{p-2} \nabla u\right)+\lambda|u|^{p-2} u=0$. Proc Am Math Soc. 109, 157-164 (1990)

15. Lindqvist, $P$ : On the equation $\operatorname{div}\left(|\nabla u|^{p-2} \nabla u\right)+\lambda|u|^{p-2} u=0$. Proc Am Math Soc. 116, 583-584 (1992)

16. Li, YX, Xie, CH: Blow-up for p-Laplacian parabolic equations. J Differential Equations. 20, 1-12 (2003)

doi:10.1186/1687-2770-2011-2

Cite this article as: Du and Li: Non-Newtonian polytropic filtration systems with nonlinear boundary conditions. Boundary Value Problems 2011 2011:2. 